\title{
Modeling of Heat and Mass Transfer in a TEC-Driven Lyophilizer
}

\author{
Zeng-Guang Yuan, Uday Hegde \\ National Center for Space Exploration Research, Cleveland, Ohio \\ Eric Litwiller \\ Enterprise Advisory Services, NASA Ames Research Center \\ Michael Flynn, John Fisher \\ NASA Ames Research Center
}

\begin{abstract}
Dewatering of wet waste during space exploration missions is important for crew safety as it stabilizes the waste. It may also be used to recover water and serve as a preconditioning step for waste compaction. A thermoelectric cooler (TEC)- driven lyophilizer is under development at NASA Ames Research Center for this purpose. It has three major components: (i) an evaporator section where water vapor sublimes from the frozen waste, (ii) a condenser section where this water vapor deposits as ice, and (iii) a TEC section which serves as a heat pump to transfer heat from the condenser to the evaporator. This paper analyses the heat and mass transfer processes in the lyophilizer in an effort to understand the ice formation behavior in the condenser. The analysis is supported by experimental observations of ice formation patterns in two different condenser units.
\end{abstract}

\section{INTRODUCTION}

This paper describes modeling efforts carried out to develop an energy efficient condenser for a lyophilization system for solid waste dewatering and recovery for space exploration missions. Wet wastes are projected to be a significant issue for these missions [1] and processing of these wastes for resource recovery can reduce overall system mass impacts. For short-term missions, solid waste stabilization and water recovery are desirable but conversion of organic waste to $\mathrm{CO}_{2}$ is not, since excess $\mathrm{CO}_{2}$ must be processed by $\mathrm{CO}_{2}$ removal systems. Dewatering wet waste provides two benefits since the water can be reused, and the dried solid that remains is biologically stable and can be stored safely. Among common drying methods, lyophilization, or freeze-drying, produces the most complete separation [2]. Solids can be dried to $1-3 \%$ water content [3], and many volatile, odor-causing compounds remain trapped in the porous solid, resulting in cleaner water. Both liquid and solid wastes can be lyophilized. The process is most appropriate for lowvolume, highly contaminated waste streams such as feces, wet trash, and concentrated wastewater.

Lyophilization is a batch or semi-batch process, consisting of the following steps:

1) Freezing - The material is frozen, separating ice crystals from contaminants.

2) Primary Drying - Moderate vacuum $(<100 \mathrm{~Pa})$ is applied, causing the ice to sublime. The water vapor condenses onto a cold surface.

3) Secondary Drying - When ice crystals have been removed, the remaining solid is heated further, vaporizing residual water.

In commercial lyophilizers, heat is moved by pumping a fluid through a vapor-compression refrigeration cycle. Traditional refrigerators can achieve high efficiencies, but they present a number of problems in this application. Refrigeration cycles that incorporate a phase change of the working fluid generally use gravity to control the fluid in its liquid phase, and there are safety and reliability issues associated with highpressure gases and compressors.

To avoid these problems - and to minimize system mass, power, and volume - thermoelectric heat pumps (TECs) are employed [4]. TECs make use of the Peltier effect to move heat directly through a semiconducting material subjected to a voltage. TECs are often regarded as less energy-efficient than compressor-based heat pumps, but when operated under relatively low heat loads and temperature gradients, efficiency increases.

Figure 1 illustrates the heat and mass transfer mechanisms that control drying in a thermoelectric 
lyophilizer. As seen in the figure, the lyophilizer consists of three primary components: (i) the evaporator where water vapor sublimes from the frozen waste, (ii) the condenser where the water vapor is condensed to ice and (iii) a TEC component which closes the loop for heat transfer between the condenser and evaporator sections.

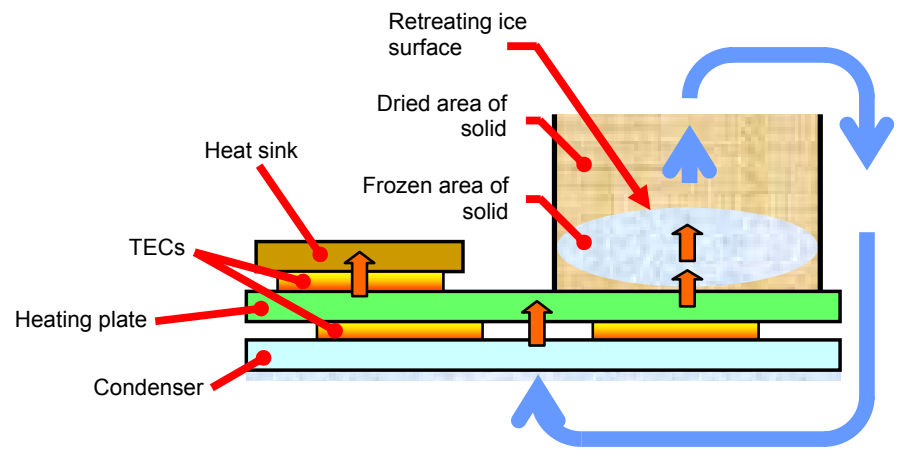

Figure 1. Vapor (blue arrows) and heat (red arrows) transfer path in the NASA Ames lyophilizer

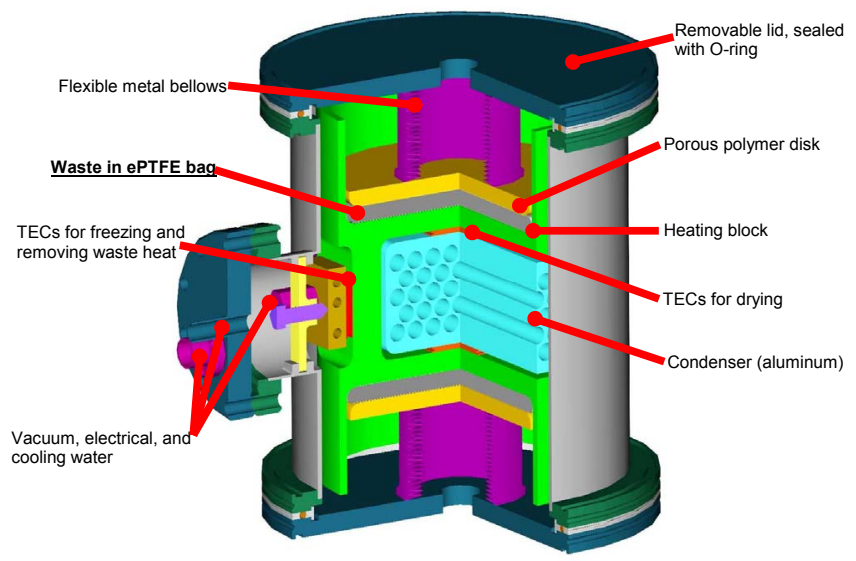

Figure 2. NASA Ames lyophilizer design

The NASA Ames design that implements this concept is shown in Figure 2. Porous bags filled with waste (gray in Figure 2) are compressed between the heating block (green) and the removable lid/bellows assemblies. The inside of the bellows is exposed to atmospheric pressure. When vacuum is applied, a force equal to 1 atmosphere times the cross-sectional area of the bellows is transmitted to the bags by the porous disks. The remainder of the force resulting from the pressure difference compresses the O-rings at the lids.

This paper focuses primarily on the condenser portion of the heat and mass transfer loop. At the condenser, heat is removed from the incoming water vapor, resulting in ice formation on the condenser surface. Since ice is a poor conductor of heat, it is advantageous to reduce the thickness of the ice layer to improve the efficiency of the heat removal process. It is clear that uniform deposition of the ice will provide the optimum conditions in this regard for given surface area of the condenser. For example, if water vapor condenses on a thicker section of ice, heat transfer to the condenser is reduced because of the reduced temperature gradient. Uneven ice formation can also impact the water removal process during the melting phase under microgravity conditions likely to be experienced during space travel.

It would appear that increasing the surface area of the condenser would also be beneficial, by reducing the condenser ice thickness for a given volume of water condensed and thereby increasing energy efficiency. This aspect is first considered from an overall closed loop analysis of the heat flows which includes the characteristic equation of the TECs. This also highlights the energy-efficient nature of the device, critical for advanced life support applications, since the heat of sublimation provided at the evaporator is recovered in the condenser and is pumped back to the evaporator to close the thermal loop. This analysis provides guidelines on the influence of TEC current, area ratio of the active evaporator and condenser surfaces, and evaporator temperature on the drying time and overall energy expenditure under uniform ice deposition conditions.

This analysis is followed by a numerical simulation of vapor transport to the condenser from the evaporator. The simulation includes the effects of non-condensable gas (NCG) in the vacuum chamber which primarily is introduced from leaks. It is shown that the NCG impacts ice formation patterns in the condenser and can lead to uneven ice formation. Experimental results on ice formation in two different condensers are shown and related to the results of the analysis.

\section{MODELING}

\section{THERMAL LOOP ANALYSIS}

\section{Assumptions}

1) Both frozen waste and condensed ice have constant, but different, cross section areas.

2) The water vapor sublimes uniformly from the frozen waste surface and deposits uniformly on the condenser side.

3) The variation of water vapor pressure and temperature in the chamber are negligible, i.e., the pressure and temperature vary with time only.

4) Quasi-steady state exists in the system.

5) TEC parameters, a, p, k and $G$ are constant.

6) The thermal properties of ice and water vapor are constant.

\section{Formulation}

During the drying process, ice moves from the frozen waste material to the condenser by sublimation. Thus the thicknesses of the ice on both sides vary with time. The electrical current fed to the TEC remains constant. (A second TEC fed from a varying current source maintains the heating block at a constant temperature.) The temperature on the condenser side of the TEC is floating and automatically determined by the process. 
The drying process can be formulated as follows:

The characteristic equation of the TEC unit is [5]

$q_{c}=2 N\left[a I T_{c}-\frac{I^{2} p}{2 G}-k\left(T_{H}-T_{c}\right) G\right]$

Under the present operation regime, only $q_{c}$ and $T_{c}$ of the TEC vary with time [4]. Thus the equation of the TEC can be rewritten as

$$
q_{c}(t)=\xi T_{c}(t)+\zeta
$$

where $\xi$ and $\zeta$ are constants defined as follows:

$$
\begin{aligned}
& \xi=2 N(a I+k G) \\
& \zeta=-2 N\left(\frac{I^{2} p}{2 G}+k G T_{H}\right)
\end{aligned}
$$

On the condenser side, the heat flux conducted through the ice must be equal to the sublimation heat released from the water vapor when it condenses. Thus,

$$
q_{c}=\theta \rho A_{c} \frac{d h_{c}(t)}{d t}
$$

Although the heat dumped to the evaporation side from the TEC is more than $q_{c}$, the amount of heat that exceeds $q_{c}$ is actually removed by the second TEC to the heat sink and then convectively to the environment. Thus, the net heat received by the frozen waste is the same as that removed from the condenser, i.e., $q_{c}$. Therefore, on the evaporation side, we have

$$
q_{c}=-\theta \rho A_{e} \frac{d h_{e}(t)}{d t}
$$

Heat conduction through the condensed ice on the condenser and the ice in the frozen waste can be described by the following two equations.

$$
\begin{aligned}
& q_{c}=\lambda A_{c} \frac{T(t)-T_{c}(t)}{h_{c}(t)} \\
& q_{c}=\lambda A_{e} \frac{T_{H}-T(t)}{h_{e}(t)}
\end{aligned}
$$

Equations (1) through (5) form a closed set for 5 unknown variables: $q_{c}(t), T(t), T_{c}(t), h_{c}(t)$ and $h_{e}(t)$. There are two first order differential equations in the system, thus we need two initial conditions to yield a special solution. At the beginning of the drying period, there is no ice on the condenser surface, thus the first initial condition is

$$
h_{c}(0)=0
$$

Also, at the initial time, all the ice is on the evaporation side, thus

$$
h_{e}(0)=\frac{V}{A_{e}}
$$

where $V$ is the total volume of ice and it is assumed that the volume of solid texture or matrix in the waste is negligible (this assumption can be removed without loss of generality). The porosity and permeability of the dry solid texture are normally large, ranging from 0.5 to 0.9 and from a fraction to a few Darcies, respectively. Thus, the effects of the solid texture on the thermal and flow process are negligible.

\section{$\underline{\text { Results }}$}

By solving Equations 1 through 7, we get the following equation for the volume of ice, $V_{c}(t)$, formed on the condenser, as a function of time.

$$
V_{c}(t)=\frac{1}{\omega}\left(\sqrt{A t+B^{2}}-B\right)
$$

where $\omega, A$ and $B$ are constants defined as follows:

$$
\begin{aligned}
\omega & =\left(\frac{A_{e}}{A_{c}}\right)^{2}-1 \\
A & =\frac{2 \lambda \omega A_{e}^{2}}{\theta \rho}\left(T_{H}+\frac{\zeta}{\xi}\right) \\
B & =V+\frac{\lambda A_{e}^{2}}{\xi}
\end{aligned}
$$

When all the ice on the waste side has moved to the condenser, we have $V_{c}(t)=V$. Applying this condition to Equation 8, we get the total time needed for drying

$$
t_{\text {total }}=\frac{\theta \rho V\left[\frac{V(a I+k G)}{\lambda A_{e}^{2}}\left(1+\frac{1}{\eta^{2}}\right)+\frac{1}{N}\right]}{I\left(2 a T_{H}-\frac{I p}{G}\right)}
$$

Equation 9 shows that for a given TEC, the total drying time is a function of the total volume of ice, $V$, the driving current to the TEC, $I$, the area of the evaporator, $A_{e}$, and the area ratio of the condenser to the evaporator, $\eta$.

For the Ames developed lyophilizer, the following parameters apply

$$
\begin{aligned}
& N=254 \\
& G=0.121 \mathrm{~cm}
\end{aligned}
$$




$$
\begin{aligned}
& T_{H}=258 \mathrm{~K} \\
& A_{e}=0.032 \mathrm{~m}^{2} \\
& V=h_{e} A_{e}=1 \mathrm{~cm}^{*} 0.032 \mathrm{~m}^{2}=0.32 \mathrm{E}-3 \mathrm{~m}^{3}
\end{aligned}
$$

The TEC parameters are chosen for $\mathrm{T}_{\text {ave }}=268 \mathrm{~K}$, [5] as

$$
\begin{aligned}
& a=0.2 \mathrm{E}-3 \mathrm{Volts} / \mathrm{K} \\
& p=0.94 \mathrm{E}-3 \mathrm{Ohm} \mathrm{cm} \\
& k=0.0179 \mathrm{~W} /(\mathrm{cm} \mathrm{K})
\end{aligned}
$$

The ice properties are [6]

$$
\begin{aligned}
\lambda & =1.9 \mathrm{~W} /(\mathrm{m} \mathrm{K}) \\
\theta & =2790 \mathrm{~kJ} / \mathrm{kg} \\
\rho & =920 \mathrm{~kg} / \mathrm{m}^{3}
\end{aligned}
$$

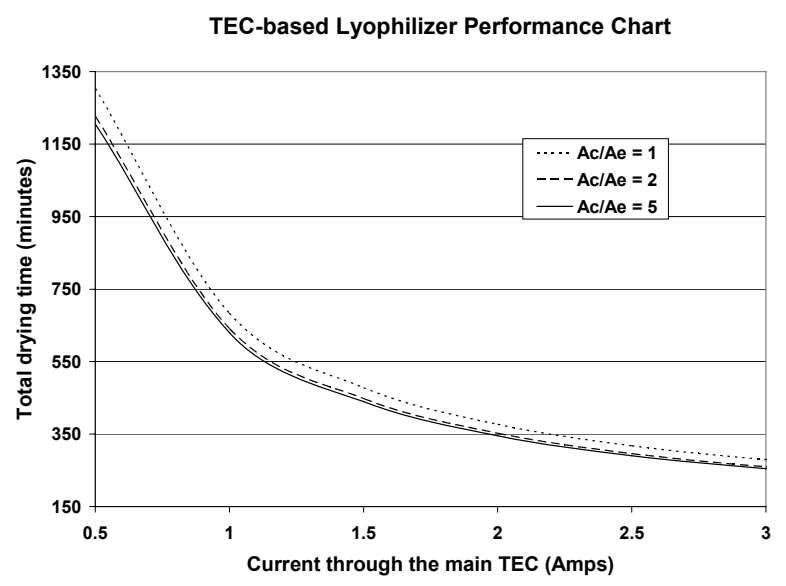

Figure 3. Total drying time as a function of TEC current

Figure 3 plots the drying time as a function of TEC current for different area ratios. These curves, in a sense, represent the performance characteristics of the lyophilizer. It is remarkable that the drying time is relatively insensitive to the area ratio for $\eta>2$. This implies that not much is gained by arbitrarily increasing this area ratio.

\section{NUMERICAL SIMULATION OF VAPOR TRANSPORT}

The impact of condenser surface area on ice formation in the presence of NCG is next considered. To this end, two-dimensional numerical simulations are performed with the flow domain represented as a channel. In a twodimensional representation, for fixed flow domain volume, increasing the surface area implies reducing the channel width for the gas flow. Two cases are therefore considered labeled (a) small channel and (b) large channel, respectively. Figure 4 provides the geometry for the small channel case. The dimensions are compatible with a condenser unit tested experimentally at NASA Ames.

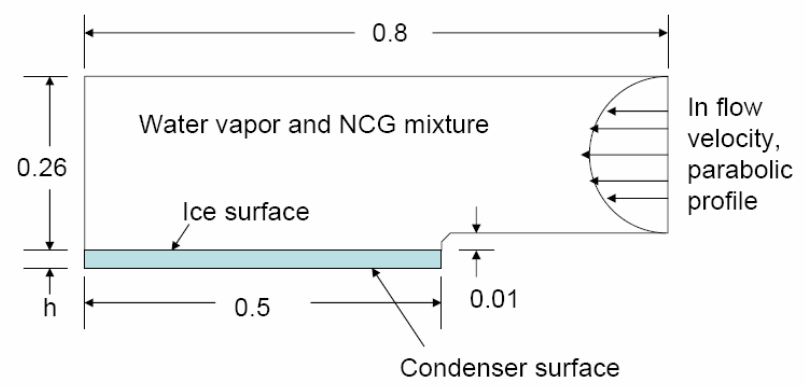

Figure 4. Geometry of the small channel condenser (all dimensions in $\mathrm{m}$ )

A parabolic gas/vapor flow is assumed to enter the channel. A thin layer of ice is assumed to have been formed on the condenser surface as shown. Only water vapor is allowed to condense on the ice surface, not NCG [7]. The temperature on the condenser surface is assumed to be constant since the conductivity of aluminum is about two orders of magnitude higher than that of ice. On the ice surface, the heat flux is coupled with mass flux by the heat of sublimation of the water vapor. A no-slip condition is imposed on the ice surface. In addition, all the boundaries, except the inflow boundary and the ice surface, of the flow domain are adiabatic, impermeable solid walls. The computational software COMSOL is used for the simulation.

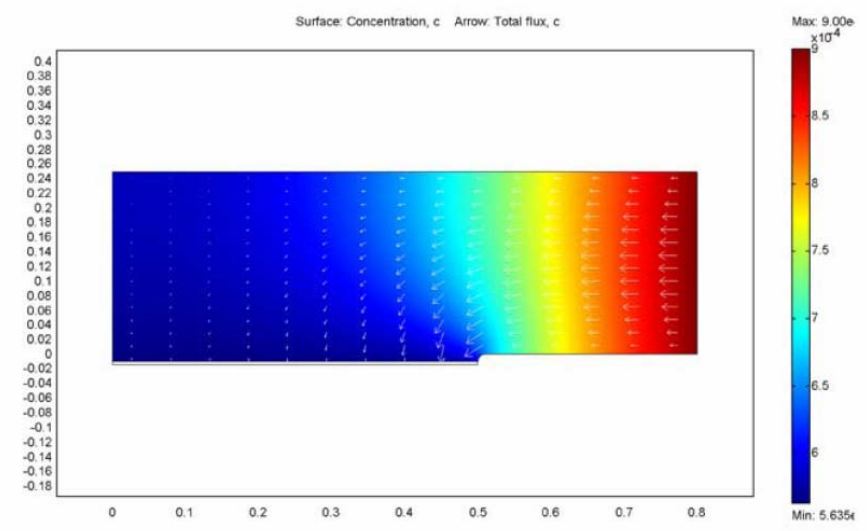

Figure 5. Water vapor concentration field and mass flux (small channel condenser)

Figure 5 shows the water vapor concentration field and mass flux. The average NCG concentration in the condenser for this case is approximately $50 \%$. It is seen from the water vapor concentration field that the concentration is high in the inlet regions and rapidly falls off toward the rear of the condenser. Consistently, the vapor mass flux condensing onto the ice layer is also high in the inlet region. It is also found that while the total pressure in the condenser is almost constant, the partial pressures of the NCG and water vapor behave in opposite manner, i.e., the partial pressure of the vapor decreases from the entrance of the condenser to the rear whereas the partial pressure of the NCG increases. 
Normalized vapor mass flux along ice surface

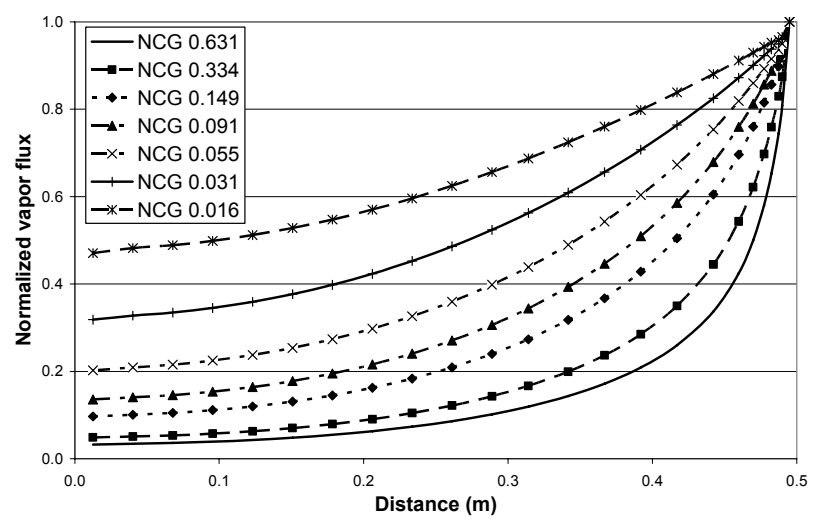

Figure 6. Normalized water vapor flux distribution for different NCG concentrations in the condenser

The impact of the NCG concentration on water vapor mass flux is shown in Figure 6. It is clearly seen that as the NCG concentration decreases the water vapor mass flux along the ice surface becomes more uniform. While not shown, it can be inferred that for the case of no NCG the water vapor mass flux profile will be flat, indicating uniform ice deposition.

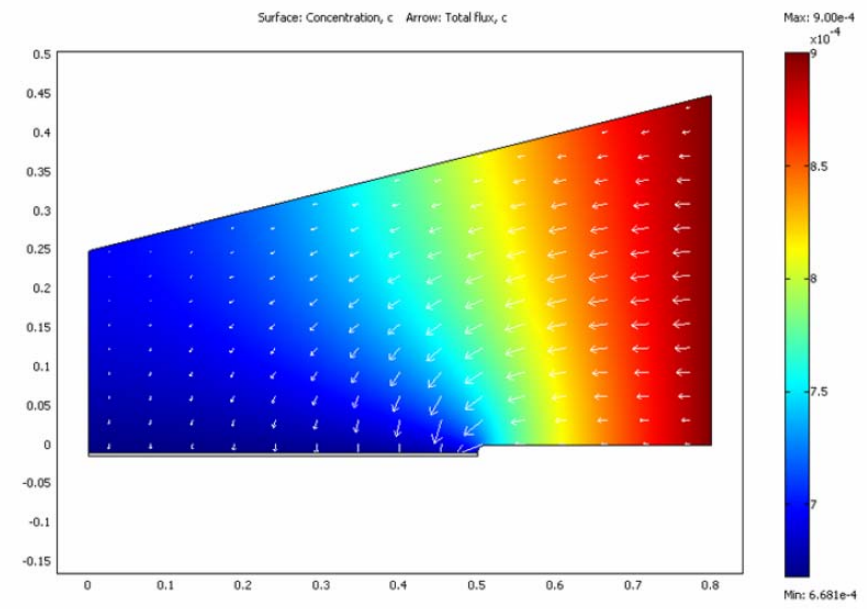

Figure 7. Water vapor concentration field and mass flux (large channel condenser)

In order to reduce the impact of NCG on the ice formation pattern, it is useful to ascertain the behavior when the channel is opened up (large channel case). Here, the inlet cross section area is increased whereas the opposite end is maintained at the same area as before. This provides a more favorable flow regime for the vapor to move further downstream. The computations (see Figure 7) show that indeed the vapor profiles extend further downstream in this case, indicating more uniform ice deposition compared to the small channel case.

\section{EXPERIMENTAL RESULTS}

Corresponding to the two condenser geometries considered in the numerical simulations, two units were fabricated and tested in the NASA Ames lyophilizer. In both cases, the area ratio of the condenser surface to the evaporating surface is more than two, as suggested by the thermal loop analysis discussed earlier. However, in the small channel case, the area ratio is about five and the fins forming the channels are closer together whereas in the large channel case, the area ratio is slightly over two and thus the fins can be further apart and tapered with a larger inlet cross sectional area.

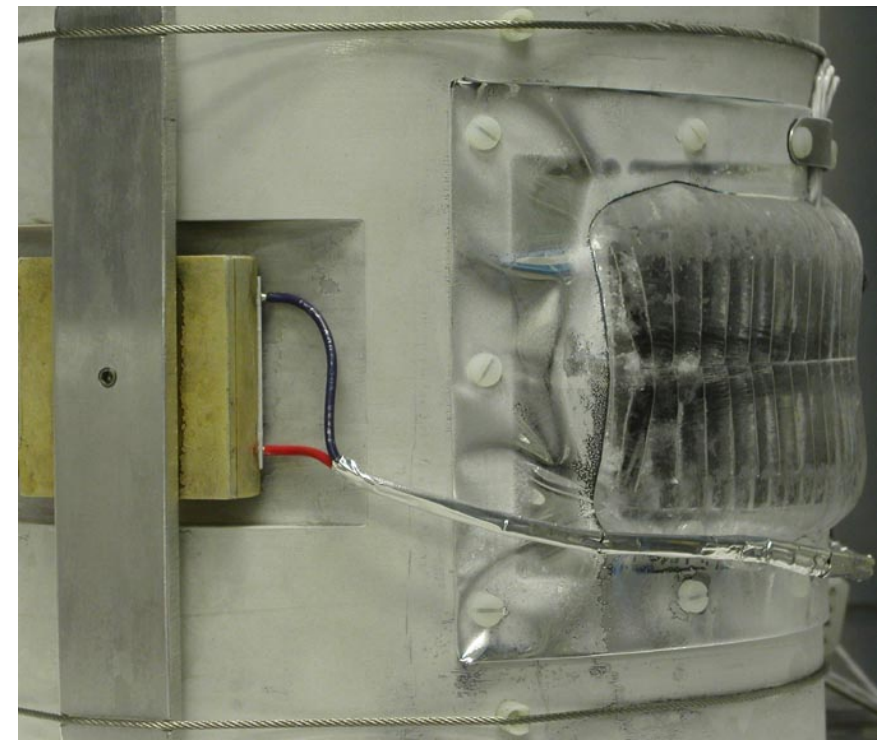

Figure 8. Small channel condenser image showing ice formation covering the inlet

An image of the ice formation for the small channel case is shown in Figure 8. It was found in this case that ice formed primarily at the entrance region of the condenser and covered it, blocking further ice deposition towards the rear of the unit. Consistent with the numerical simulation, it appears that the presence of NCG reduces the partial pressure of the vapor towards the rear of the condenser, resulting in preferential ice formation towards the front.

For the large channel case, the ice formation is found to be significantly more uniform as shown in Figure 9. This is also consistent with the numerical simulation results, which indicate that the partial pressure of the vapor is more uniform in this case. 


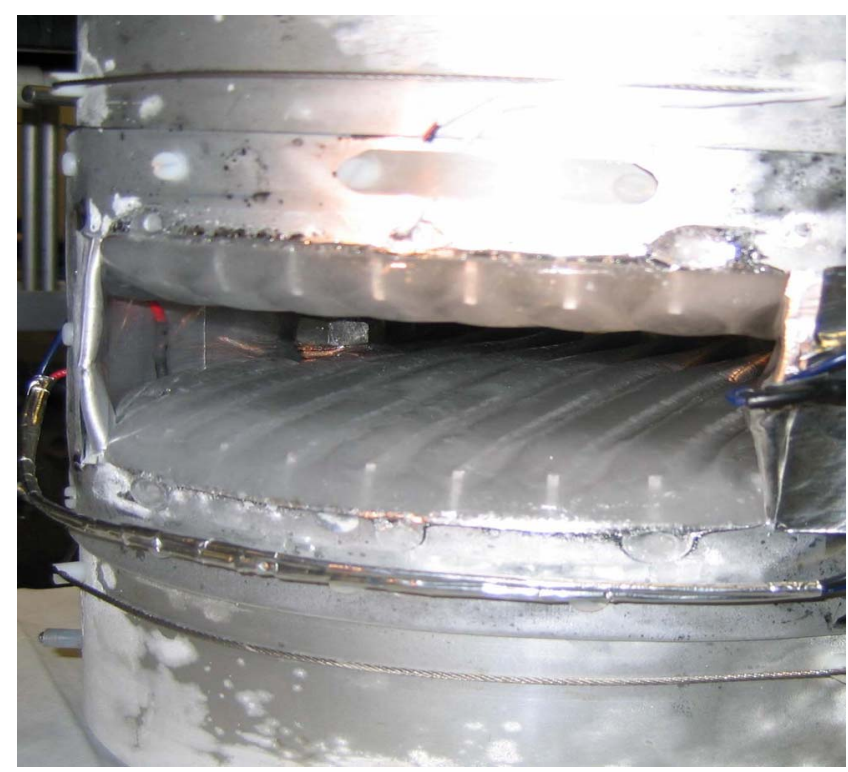

Figure 9. Large channel condenser image showing uniform ice deposition

\section{CONCLUSION}

Heat and mass transfer in a TEC-driven lyophilizer has been analyzed. A closed loop formulation of the thermal path shows that the drying time is not very sensitive to the condenser surface area to evaporator surface area ratio as long as this value is greater than two. At the same time, numerical simulations of the vapor flow in the presence of NCG indicate significant non-uniformity of the water vapor mass flux on the condenser surface particularly for large values of the condenser surface area ratio for given condenser volume. Reducing the condenser surface area while maintaining a condenser/evaporator ratio of at least two results in a satisfactory scenario where the vapor mass flux and corresponding ice layer thickness on the condenser surface become more uniform. This is beneficial from the viewpoint of increased energy efficiency while maintaining reasonable drying times. The results of the analysis are confirmed from experimental results with two condensers with larger area ratio (larger number of smaller channels) and smaller area ratio (smaller number of larger channels).

\section{ACKNOWLEDGMENTS}

Zeng-Guang Yuan and Uday Hegde were supported by NASA Glenn Research Center under Cooperative Agreement NCC3-975. Also at NASA Glenn, John Caruso and John Snead served as project managers and Daryl Edwards supported engineering design and manufacturing. Useful discussions were held with Charles Neiderhaus of NASA Glenn.

\section{REFERENCES}

1. Kaplan, D. L. and Hoffman, S. J., Human Exploration of Mars: The Reference Mission of the NASA Mars Exploration Study Team, http://wwwsn.jsc.nasa.gov/marsref//toc.pdf, 1997.

2. Coumans, W. J., Kerkhof, P., and Bruin, S., Theoretical and Practical Aspects of Aroma Retention in Spray-Drying. Drying Technology, 1994. 12 (1-2): pp. 99-149.

3. Liapis, A. I., Freeze Drying in Handbook of Industrial Drying, A. S. Majumdar, Editor, 1989, Marcel Dekker: New York. Pp. 295-326.

4. Litwiller. E., Reinhard, M., Flynn, M., and Fisher, J., Lyophilization for Water Recovery, SAE Paper 200201-2348.

5. Melcor Corporation, Frigichip(R) Thermoelectric Cooling Devices, 1994.

6. CRC Press, Handbook of Chemistry and Physics, $84^{\text {th }}$ ed., 2003.

7. Roshenow, W., and Choi, H., Heat, Mass, and Momentum Transfer, Prentice Hall, pp. 397-400, 1961.

\section{CONTACT}

Zeng-Guan Yuan, Ph.D., P.E.

Zengguang.Yuan@grc.nasa.gov

\section{DEFINITIONS, ACRONYMS, ABBREVIATIONS}

\begin{tabular}{|c|c|}
\hline a: & Seebeck coefficient (Volt/Kelvin) \\
\hline $\mathbf{A}_{\mathrm{c}}$ : & Area of condensing surface $\left(\mathrm{m}^{2}\right)$ \\
\hline$A_{e}:$ & Area of evaporation surface $\left(\mathrm{m}^{2}\right)$ \\
\hline G: & Area/length of TEC element $(\mathrm{cm})$ \\
\hline $\mathbf{h}_{\mathbf{c}}:$ & Ice thickness on condenser side (m) \\
\hline $\mathbf{h}_{\mathbf{e}}$ : & Ice thickness on evaporation side (m) \\
\hline I: & Current (Ampere) \\
\hline k: & Thermal conductivity (Watt/cm/Kelvin) \\
\hline N: & Number of TEC elements \\
\hline NCG: & Non-condensable gas \\
\hline p: & Resistivity (Ohm-cm) \\
\hline Pa: & Pascals (Newton $/ \mathrm{m}^{2}$ ) \\
\hline $\mathbf{q}_{\mathbf{c}}:$ & Heat flux from cold side (Watt) \\
\hline t: & Time (Second) \\
\hline T: & Temperature in chamber (Kelvin) \\
\hline $\mathrm{T}_{\mathrm{c}}:$ & Temperature on cold side (Kelvin) \\
\hline $\mathbf{T}_{\mathrm{H}}:$ & Temperature on hot side (Kelvin) \\
\hline TEC: & Thermoelectric cooler \\
\hline V: & Total volume of ice $\left(\mathrm{m}^{3}\right)$ \\
\hline$\theta:$ & Sublimation heat of ice $(\mathrm{kJ} / \mathrm{kg})$ \\
\hline$\rho:$ & Density of ice $\left(\mathrm{kg} / \mathrm{m}^{3}\right)$ \\
\hline$\lambda:$ & Thermal conductivity of ice (Watt/m/Kelvin) \\
\hline$\xi:$ & Constant, defined in Eq. 1a (Watt/Kelvin) \\
\hline$\zeta:$ & Constant, defined in Eq. 2a (Watt) \\
\hline$\omega:$ & TEC parameter defined by Eq. 8 \\
\hline$\eta:$ & \\
\hline
\end{tabular}

